\title{
An illuminating experience
}

\author{
Michael Eisenstein
}

New methods are enabling neuroscientists to apply optogenetics to big brains.

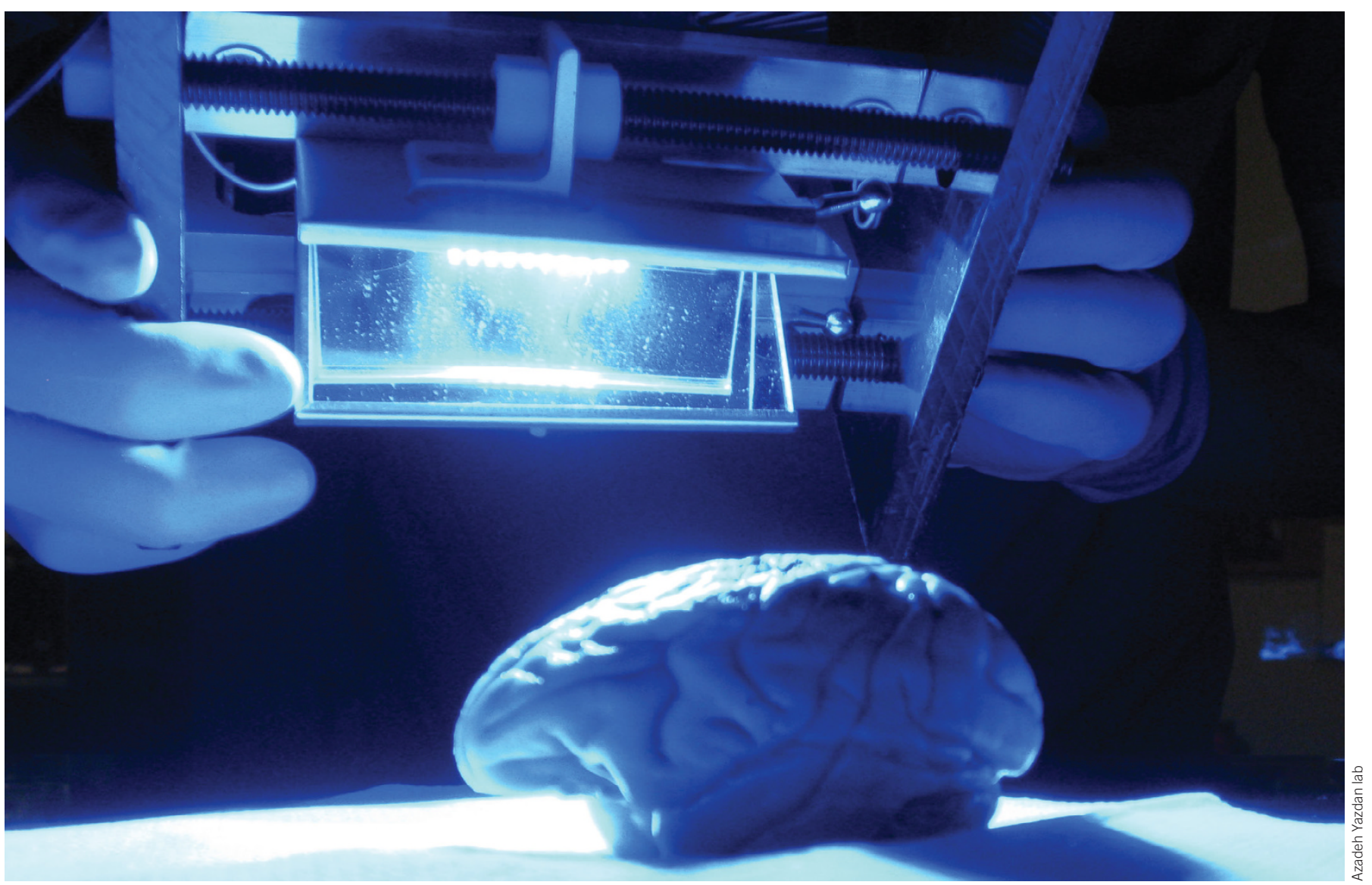

The concept of optogenetics sounds almost too good to be true-making direct manipulation of brain activity literally as simple as turning on a light. But scientists have been using laser illumination to selectively activate and deactivate genetically-modified neurons in living rodents for more than a decade now. Armed with this tool, it has become possible to directly link individual brain cell populations and circuits with functions

Michael Eisenstein is a freelance science writer in Philadelphia. ranging from eating and sleeping to memory and social behavior, and to dissect the neurological effects of diverse diseases.

But more difficult research problems require more sophisticated models, as Azadeh Yazdan learned while researching stroke rehabilitation as a graduate student. "That project failed because it went from rodent models to humans," says Yazdan, now a neuroengineering researcher at the University of Washington. "They're good models to study a lot of things, but the cortical anatomy is so different from humans." Accordingly, there has been considerable interest in porting the optogenetics toolbox to closer evolutionary relatives.

Edward Boyden, who helped develop optogenetics with postdoctoral mentor Karl Deisseroth at Stanford University, spearheaded the push to apply the method in nonhuman primates (NHPs) upon starting his own lab at MIT in 2008. A year later, his group published the first study showing that the same techniques that worked in rodents could be translated into macaques ${ }^{1}$. Progress since then has occurred in fits and starts. "A rodent 


\section{TECHNOLOGY FEATURE}

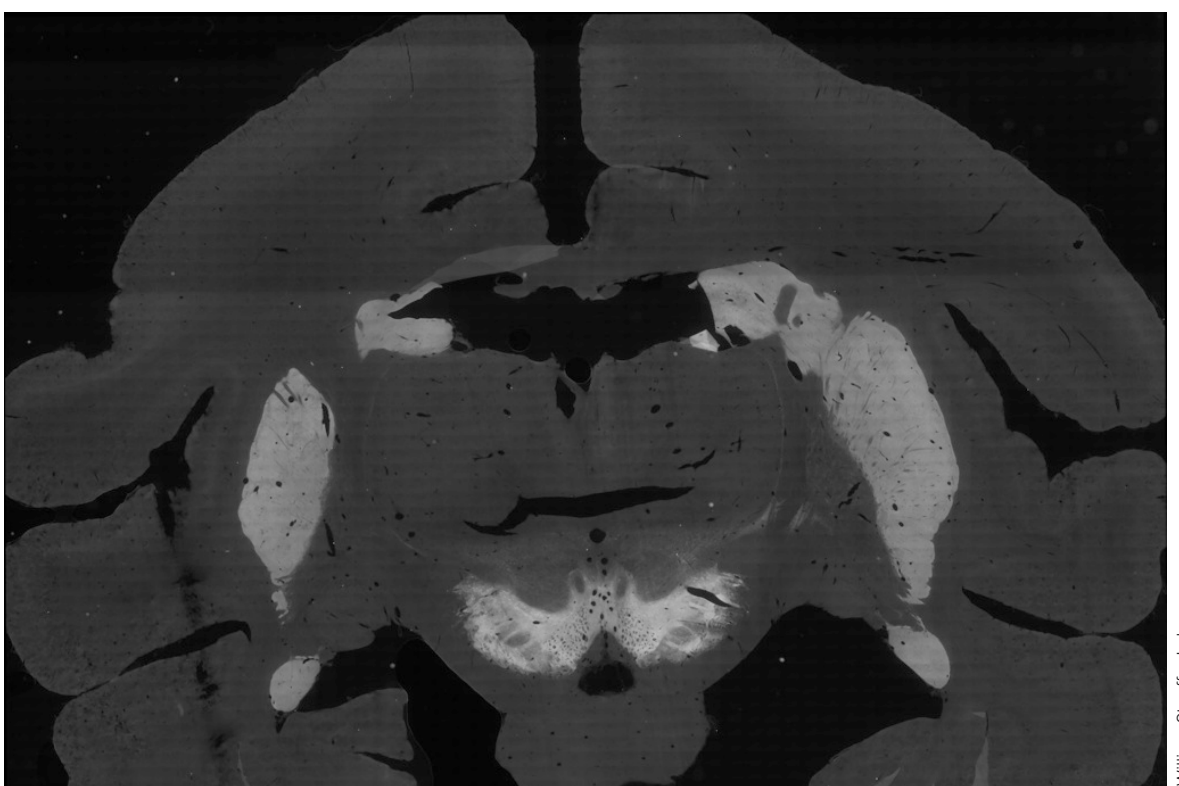

DOPE IMAGE | William Stauffer and colleagues used optogenetics to manipulate the dopamineresponsive reward system of the monkey brain, shown here as fluorescently-labeled neurons within a tissue section.

experimenter might run 100 mice in a study per year, versus two monkeys for five years," says Boyden. "It's an intrinsically slower process."

But the pioneers in this space are now hitting pay dirt. A growing number of studies have demonstrated that lightbased manipulation of neurons can have measurable effects on NHP behavior and neurological function. For example, University of Pittsburgh researcher William Stauffer and colleagues showed they could influence monkeys during a behavioral task by selectively illuminating neurons that produce the 'feel-good' neurotransmitter dopamine ${ }^{2}$. And although primate optogenetics is still in its infancy and many technical challenges remain, neuroscientists now see unprecedented opportunities to explore the mysteries of brain function in an almost-human context.

\section{The guiding light}

If you are seeing these words, it is because proteins in your retina known as 'opsins' are reacting directly to patterns of light and transmitting that information to the brain's visual centers. Optogenetics relies on a specific set of opsin proteins that have been selected based on the wavelengths of light to which they respond. For example, channelrhodopsin-2 is an engineered opsin derived from algae that reacts to blue light. It forms a channel that is normally closed off, but blue light causes it to open up temporarily. If neurons are genetically modified to express this protein, the lightinduced opening of that channel allows ions to flow through the cell membrane, creating conditions that cause that neuron to fire.

This genetic modification can be achieved by packaging the opsin gene into a virus, and then injecting the virus into the part of the brain one intends to investigate. Once brain cells take up the viral DNA, they will begin to produce the opsin protein and can then be manipulated by shining a laser through a thin fiber-optic probe. Channelrhodopsin-2 acts as an 'on switch' for neurons, but there are also other opsins that enable selective inhibition of brain cells and circuits.

The concept was instantly appealing to those who had toiled for years with more primitive tools. "As soon as it came out, I thought 'We've got to get this," says Gregory Horwitz, a behavioral neuroscientist at the University of Washington. Before optogenetics, most functional neuroscience studies in NHPs used electrodes to deliver targeted jolts to activate different brain structures-an approach that can be highly effective, but is also something of a blunt instrument. "You don't know exactly what's happening when you deliver current to a local area of the brain," says David Sheinberg, who studies sensory perception at Brown University. By putting neurons under the control of genetically-controlled markers and precisely timed pulses of light, it now became feasible to dictate the time and location of activation and inhibition with much greater precision.

And, importantly, many of the core techniques required to perform optogenetics in NHPs were already well established. For example, researchers have been using viruses belonging to the adeno-associated virus (AAV) family to deliver genes to the primate brain for decades-indeed, this vector has even been used in humans for numerous gene therapy clinical trials. Likewise, the surgical procedures used for electrophysiology could easily be adapted for the implantation of optical probes, or 'optrodes', that allow researchers to both illuminate neurons of interest and detect the resulting electrical activity they produce.

In the three years following the Boyden group's pioneering demonstration, other labs reported success in using AAV to deliver opsin genes into the NHP brain, generating neuron populations that could be reliably manipulated by light. But these experiments initially offered little value beyond proof of concept. "In rodent studies, there were many examples of behavior being modulated with opsins, but people could not really get a solid behavioral change in monkeys," says Adriana Galvan, a neuroscientist at Emory University.

\section{Volume control}

Several obstacles have made it challenging to take NHP optogenetics to the next level. The most obvious is size; for example, the macaque brain contains roughly 100 times as many neurons as the mouse brain. "It's a question of volume," says Galvan. "You need to reach a larger number of neurons in the primate brain to see a similar change in behavior like you would in rodents."

Injections of AAV do not spread far in the brain, which means only small numbers of neurons will receive the opsin gene. This makes multiple doses necessary to effect a meaningful response. "If you want 


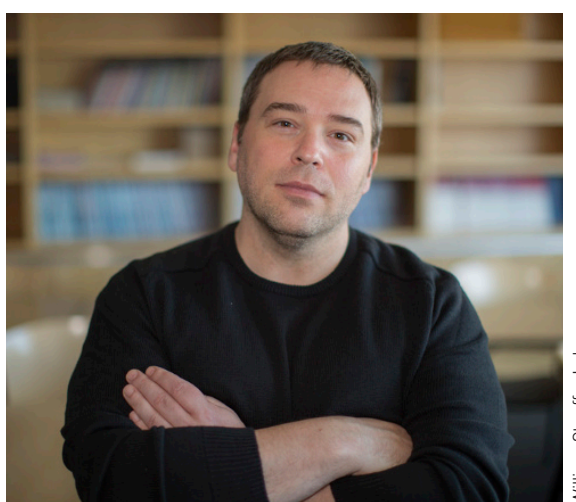

CRACKING BIG CIRCUITS | William Stauffer, neuroscientist at University of Pittsburgh, uses optogenetics in primates to understand neural mechanisms of reward processing.

to cover the motor or somatosensory area of a monkey brain, you have to do tens or even hundreds of injections," says Yazdan. Several groups have achieved success in this fashion, but she notes that such large numbers of injections can require multiple day-long sessions and increase the risk of damaging the brain. As an alternative, her team has employed an approach in which large volumes of virus are delivered into the brain, allowing them to spread evenly across much bigger swaths of tissue ${ }^{3}$. Using this approach, Yazdan's group can introduce opsin into square-centimeterscale patches of cortex with 70-80\% efficiency and minimal tissue damage.

The flip side of this problem is that it can also be challenging to deliver adequate light to these larger volumes of opsin-expressing neurons. In the first successful demonstration of optogenetically-modified behavior in NHPs, Wim Vanduffel's team at KU Leuven Medical School shed more light on the problem by using two fiber-optic probes rather than one $e^{4}$. Alternative probe designs are also proving useful. Whereas most groups have uses fiber optics that just emit light at their tip, Leah Acker of Duke University and Robert Desimone at MIT developed 'omnidirectional' probes that spray light in every direction, simultaneously illuminating volumes of up to 10 cubic millimeters ${ }^{5}$. Yazdan's team has been able to illuminate even larger areas of the brain by embedding semi-transparent arrays of electrodes under a clear 'window' in the skull. This makes it possible to optogenetically manipulate many patches of neurons in parallel ${ }^{3}$. "We could record from about 2 $\mathrm{cm}^{2}$ of cortex, with the ability to record and maintain optical access for simultaneous stimulation," she says.

Brain tissue is densely crowded, and some light wavelengths travel through it better than others. Unfortunately, the blue light that activates channelrhodopsin-2 is not best suited for this voyage, as it tends to scatter. As a solution, Boyden's group and others have engineered alternative opsins that respond to red light, expanding the radius of activation that can be achieved with optogenetic experiments. "It goes deeper into the brain than other colors because it's the one color that blood does not absorb," says Boyden, noting that his collaborators have used one such opsin to modulate visual behavior in macaques by manipulating cells deep within the cerebral cortex ${ }^{5}$.

Even well-defined structures in the primate brain can contain a host of different types of neurons, which react to distinct chemical signals. In some cases, one can rely on the physical 'wiring' of neurons to decide which cells to target and which cells to monitor, but there has also been a lot of effort to develop genetic strategies for limiting opsin expression to select subsets of cells. For example, one can tether opsin genes to cell type-specific regulatory DNA sequences known as promoters. One can only cram so much DNA into an AAV particle, however, and so researchers have had to get creative. When Stauffer decided that he wanted to selectively manipulate only neurons that synthesize and release the neurotransmitter dopamine, he collaborated with Boyden to develop a two-virus, cell-specific optogenetic system ${ }^{1}$. One carries the inactive opsin gene, which is only turned on in the presence of a second, 'activator' virus powered by a dopamine neuron-specific promoter. As a result, his team was able to achieve remarkably specific optogenetic manipulation of this one neuronal subtype.

\section{Bright ideas}

With such tricks and tools at hand, a growing number of researchers have successfully used optogenetics to elicit specific
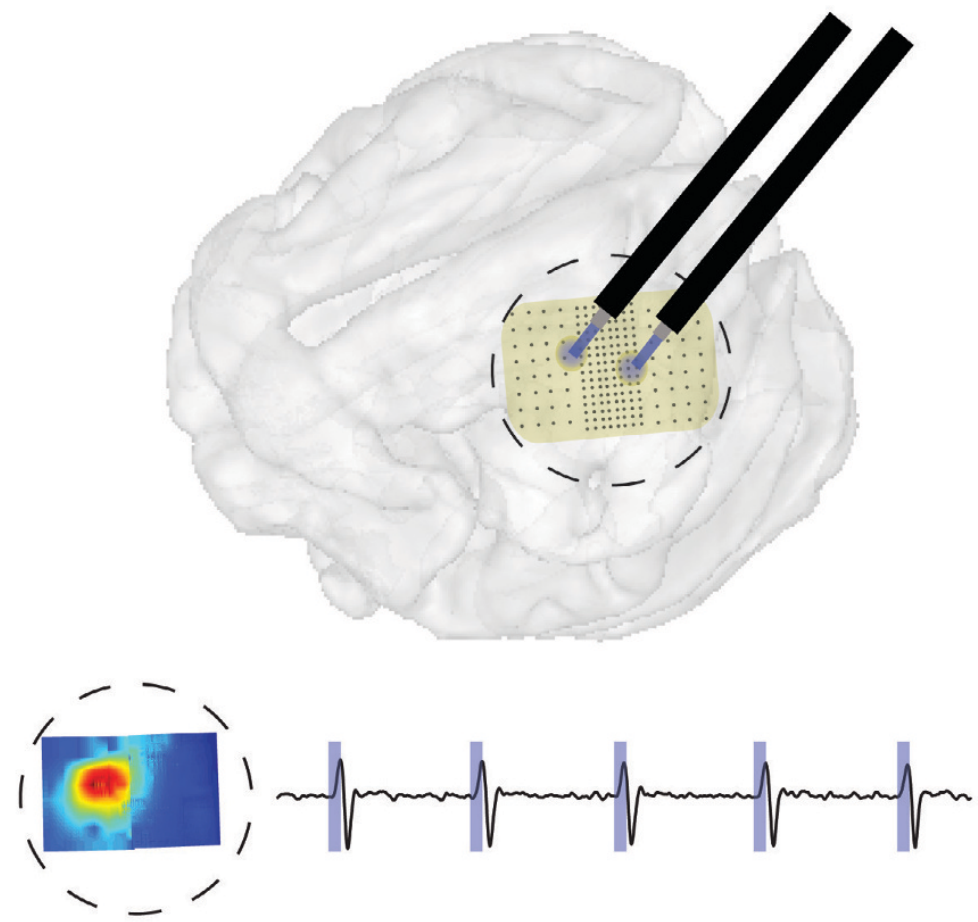

MANIPULATE AND LISTEN | Schematic of an optogenetic interface for NHPs that enables stable, large-scale stimulation and recording across the cortex (top). Examples of spatial (bottom left) and temporal (bottom right: blue rectangles represent the duration of light stimulation) light-evoked neural responses. 
behavioral effects in monkeys. These are not dramatic-but according to Sheinberg, there has been growing recognition that mirroring the powerful effects seen in rodent optogenetics is somewhat unrealistic, and perhaps not even desirable. "One of the reasons we use primates as a model is because they are capable of pretty sophisticated behavioral experiments - not so different from what people can do," he says.

Many experiments use a rapid movement of the eyes known as 'saccade' as a simple and obvious readout for optogenetic manipulation. Early studies, such as the 2012 study by Vanduffel and colleagues ${ }^{4}$, directly targeted areas of the brain that control eye movement, demonstrating that these neurons could be manipulated to affect the rate of saccade. Since then, other groups have trained primates to perform more sophisticated behavioral tests based on saccades. For example, Stauffer and colleagues showed the importance of dopamine neurons as a 'reward' system that reinforces pleasurable behaviors. They found that monkeys were much more likely to perform a visual task associated with a sweet treat when it was coupled with selective activation of these brain cells ${ }^{1}$. "The animals were clearly biased toward the option associated with optical stimulation, and that was really cool to see," says Stauffer. Sheinberg and his Brown colleague Arto Nurmikko have also used saccades as a readout for optogenetics experiments targeting the neurons in the somatosensory cortex that convey touch sensation, training monkeys to shift their gaze in response to a light-triggered virtual 'poke' on the paw'.

Yazdan and colleagues have found that they can use light stimulation to actively modify the neuronal circuits between the motor and somatosensory cortices, which respectively control movement and touch perception. "We have demonstrated that we can increase the connectivity between these two areas," she says. "People have shown that electrical stimulation can change connectivity within an area of the brain, but nobody had shown you could change it across areas at this large scale." Given her long-standing interest in finding ways to repair neurological damage, she is now exploring the possibility that this kind of targeted stimulation could one day be used in patients to repair the effects of stroke or other brain-injuring disorders. "If someone is unable to move their arm, can we go to some other area of the brain and rewire that area to recover the lost function?" she says.

The idea of using optogenetics in humans is not inconceivable, but given the rate of progress in NHPs to date, it seems more likely that the near-term clinical payoffs will be in terms of homing in on brain regions that can be treated with conventional approaches. For example, Galvan's team is using optogenetics to explore how the neurological processes that control movement break down in primate models of Parkinson's disease. "We are going to learn which circuits are working and which ones are altered," she says, "and the hope is that we'll be able to use these to develop better therapies, perhaps drugs or variants of deep brain stimulation."

The ability to perform functional brainmapping experiments in the highly-evolved primate brain promises to give researchers insights into processes like memory, learning, and sensory perception with a level of sophistication that would be all but impossible in rodents. For example, Sheinberg is studying the circuits in the brain that selectively focus on visual information like color and shape, and how that information is subsequently coupled with input from other senses-such as how one is able to 'visualize' a cube held in their hand without actually looking at it. And Stauffer is digging deeper into the role of dopamine neuron signaling in various areas of the cortex that process sensory information and decision-making, circuits that expanded considerably during the evolution of our primate cousins. "These are really important questions that optogenetics will allow us to answer," says Stauffer.

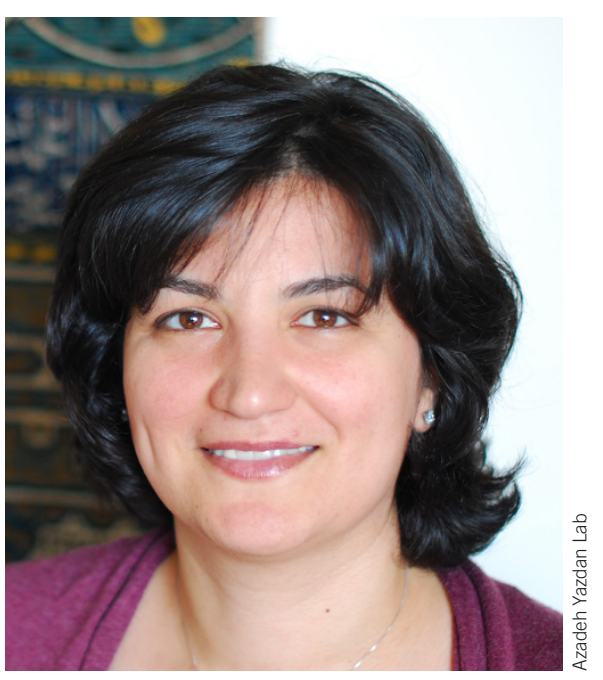

BRIDGING THE GAP | Azadeh Yazdan, a neuroengineer at University of Washington, uses optogenetics and novel recording devices in NHPs to understand plasticity in sensorimotor connections, which she hopes will help lead to new therapeutic interventions in humans with stroke.

The good news is that there is now a solid foundation of expertise for labs looking to explore this world, and early adopters are generally happy to lend a hand. "I'm consulting with two labs that didn't have any prior experience with optogenetics, but had been doing NHP electrophysiology for some time," says Yazdan. "Overall, I don't think there's a huge barrier to entry." And although labs like Boyden's are continuing to focus on pushing the technological capabilities of the field, one need not operate at the bleeding edge to benefit. "It's a tool in the toolbox - we're not going to turn into an 'optogenetics lab," says Sheinberg. "It's just something we use when it makes sense to help our science."

1. Han, X. et al. Neuron 62, 191-198 (2009).

2. Stauffer, W.R. et al. Cell 166, 1564-1571 (2016).

3. Yazdan, A. et al. Neuron 89, 927-939 (2016).

4. Gerits, A. et al. Curr. Biol. 22, 1722-1726 (2012).

5. Acker, L. et al. Proc. Natl. Acad. Sci. USA 113, E7297-E7306 (2016).

6. May, T. et al. PLoS One 9, e114529 (2014). 